LIVER

\title{
Benefit of interferon therapy in hepatocellular carcinoma prevention for individual patients with chronic hepatitis $C$
}

\author{
H Yoshida, R Tateishi, Y Arakawa, M Sata, S Fujiyama, S Nishiguchi, H Ishibashi, G Yamada, \\ O Yokosuka, Y Shiratori, M Omata
}

See end of article for authors' affiliations

Correspondence to:

Dr H Yoshida, Department

of Gastroenterology,

University of Tokyo

Hospital, 7-3-1 Hongo,

Bunkyo-Ku, Tokyo

113-8655, Japan;

yoshida-2im@

h.u-tokyo.ac.jp

Accepted for publication 20 October 2003
Background: An increase in the incidence of hepatocellular carcinoma (HCC) in Japan since the 1980s suggests an imminent outbreak in other countries where viral spread occurred more recently. Interferon therapy for chronic hepatitis $C$, in general, has been shown to prevent HCC.

Aims: To determine the scale of benefit in individual patients.

Subjects: Histologically proven chronic hepatitis $C$ patients in the Inhibition of Hepatocarcinogenesis by Interferon Therapy (IHIT) cohort (Ann Intern Med 1999;131:174), as updated in March 2003.

Methods: The lifetime risk for HCC was calculated based on HCC incidence rates, stratified by sex, age, fibrosis stage, and outcome of interferon therapy. The gain in HCC free survival was defined as the difference between expected HCC free survival with sustained virological response and that without.

Results: The gain in HCC free survival was greater when a patient was younger and fibrosis was more advanced. For example, a 30 year old male with F3 fibrosis gained 12.4 years by attaining sustained response while a patient with $\mathrm{Fl}$ fibrosis older than 60 years gained less than one year. For a treatment protocol with a given sustained response rate, prior estimation of the gain can be obtained by multiplying the calculated HCC free survival for responders by the response rate.

Conclusions: The gain in HCC free survival may serve as an indicator of the benefit of interferon therapy in terms of HCC prevention and be useful in the consideration of indication and selection of treatment protocol for individual patients.
$\mathrm{V}$ arious studies have indicated that a nationwide spread of hepatitis C virus (HCV) took place in Japan in the 1950s and 1960s. ${ }^{12}$ As a result, there has been a rapid increase in hepatocellular carcinoma (HCC) incidence since 1980, now claiming more than 30000 deaths each year. Simultaneously, there was a decline in the number of deaths assigned to cirrhosis. The decline may be partly due to advances in HCC diagnosis but the major cause seems to lie in the increasing risk of HCC as patients are getting older. The time lag of 30 years between the peaks of infection spread and HCC incidence corresponds to the observed interval between the time of blood transfusion and carcinogenesis in typical HCC patients. ${ }^{3}$ These data strongly suggest an imminent outbreak of HCC incidence in other countries, including the USA, where HCV infection is thought to have been spread more recently. ${ }^{45}$

Shortly after the discovery of HCV in $1989,{ }^{67}$ interferon therapy was confirmed to be effective against HCV infection. $^{8-10}$ We and other groups have shown that interferon therapy significantly reduces the risk of HCC development among chronic hepatitis $C$ patients. ${ }^{11-15}$ In our previous study, $50 \%$ relative risk reduction, compared with untreated patients, was observed for conventional interferon monotherapy that showed an overall sustained virological response (SVR) rate of $33 \%$, and a relative risk reduction to $20 \%$ was revealed among patients who achieved SVR. ${ }^{15}$ As the antiviral efficacy of interferon therapy has been improved by recent advances such as combination with ribavirin ${ }^{16-18}$ and introduction of pegylated interferons, ${ }^{19-22}$ we can expect that the efficacy on HCC prevention has also been strengthened.

We have also shown that cirrhosis gradually resolves once SVR is achieved, ${ }^{23}$ suggesting that interferon therapy will also prevent death due to liver failure or variceal rupture. However, HCC is clearly the dominant cause of death in patients with chronic hepatitis $\mathrm{C}$, at least among our cohort in Japan where the average age is over 50 years and most patients abstain from heavy alcohol consumption. ${ }^{24}$ Thus we have focused on HCC prevention as the primary object of interferon therapy.

Considering the current status of therapeutics, interferon therapy is clearly recommended only in a selected group of patients. ${ }^{25}$ Since the benefit of antiviral therapy differs among individual patients, the indication as well as the choice of regimen should be decided based on the expected benefit for each patient. Quantification of benefit requires reasonable assessment of the lifetime risk of HCC and the expected reduction in it with treatment. In this study, we propose an indicator, the gain in HCC free survival, to quantify the benefit specific to individual patients. The value is calculated based on both life expectancy and individualised risk of HCC, and applicable to distinct protocols with varying efficacy. It may serve as the gold standard for the benefit of antiviral therapy in terms of HCC prevention.

\section{MATERIALS AND METHODS}

\section{Incidence rates of HCC}

Crude data were obtained from the IHIT (Inhibition of Hepatocarcinogenesis by Interferon Therapy) database,,$^{15} 2324$ as updated on 31 March 2003. Every patient underwent liver biopsy in 1990 or later, and liver fibrosis was staged according to the classification system of Desmet and colleagues. ${ }^{26}$ Patients had no history of HCC, and were positive for HCV antibody and negative for hepatitis B surface antigen. We excluded those who developed HCC or dropped out of surveillance within one year after liver biopsy, and the start

Abbreviations: $\mathrm{HCV}$, hepatitis $\mathrm{C}$ virus; $\mathrm{HCC}$, hepatocellular carcinoma; SVR, sustained virological response; NNT, number needed to treat 
of observation was set at exactly one year after liver biopsy. Entry into the cohort was closed in 1999. The cohort population analysed in this study consisted of 2392 patients who received interferon monotherapy within one year of liver biopsy, and 395 patients who did not. Among 2392 interferon treated patients, $836(34.9 \%)$ showed SVR, as determined six months after cessation of interferon administration. After undergoing liver biopsy, $90 \%$ of patients abstained from alcohol except for infrequent social occasions, and only $2 \%$ continued drinking alcohol (>80 g daily).

Patients underwent abdominal ultrasonography every 3-6 months, and contrast enhanced computed tomography was also performed every 6-12 months in patients with advanced fibrosis. A final diagnosis was made based on haemodynamic patterns on contrast enhanced computed tomography, abdominal angiography, or computed tomography during angiography. Ultrasound guided tumour biopsy was performed in ambiguous cases. The SVR group showed 27 events of HCC development during an observation period of 4767 person years; in the non-SVR group, 214 events in 9922 person years; and in the untreated group, 67 events in 2168 person years.

HCC incidence rates, stratified by age, sex, and fibrosis stage at entry, were calculated in each group by the person year method. Risk ratios were analysed using Cox proportional hazard regression. Age, as ranked by 10 years, and fibrosis stage were represented by dummy variables in the analysis. Adjusted HCC incidence rates were calculated so that the sum of squares of differences between the adjusted and observed values, weighted by the number of patients in each category, was minimised while conserving the risk ratios obtained by proportional hazard regression.

\section{HCC free survival}

The probability that a patient remains free of HCC at $n$th year of observation was calculated as:

$$
\begin{aligned}
& (1-Q 1)(1-P 1) \times(1-Q 2)(1-P 2) \times(1-Q 3) \times \\
& (1-P 3) \times \ldots \times(1-Q n)(1-P n)
\end{aligned}
$$

where $Q i$ is the age and sex specific death rate in the general population and $P i$ is the annual incidence of HCC specific to the patient in the $i$ th year. Age and sex specific death rates were those published by the Ministry of Health, Welfare, and Labour of Japan for the vital statistics surveyed in $2000 .{ }^{27}$ The gain in HCC free survival by interferon therapy was defined as the area between the cumulative HCC free survival curves. This model is based on an assumption that fibrosis stage remains constant with time (see model limitations in the results section).

\section{Statistics}

Values are expressed as mean (SD) unless otherwise specified. All statistical analyses were performed with SAS Software version 6.12. We used an original program coded in Object Pascal to calculate cumulative HCC free survival.

\section{RESULTS}

\section{Incidence rates of HCC}

Demographic data of the patients analysed in the current study are summarised in table 1 , and observed HCC development and deaths are shown in table 2, illustrating that HCC was the major sequela among the cohort. Crude incidences of HCC did not differ between the untreated group and the non-SVR group in the corresponding category (data not shown). Patients with advanced fibrosis (stages F3 or F4) in the non-SVR group or in the untreated group showed a very high incidence rate. In fact, values obtained were greater than those found in $1999,{ }^{15}$ suggesting that the risk of HCC has increased with time. Fibrosis stage was determined at the time of liver biopsy and had possibly progressed during the observation period. As previously described, HCC incidence rates were substantially lower in the SVR group.

Cox proportional hazard regression analysis revealed that male sex, older age, and advanced fibrosis were associated with a higher risk of HCC, both in the non-SVR groups (table 3) and in the untreated group (data not shown). Multivariate analysis showed that the risk ratio of non-SVR to no treatment was 0.835 (95\% confidence interval (CI) $0.625-1.1125 ; p=0.2214)$. We previously showed that the risk of HCC was decreased in patients who showed normalised serum alanine aminotransferase levels in spite of continued viraemia after interferon therapy. ${ }^{24}$ However, active hepatitis recrudesces not infrequently in a short period $^{28}$ and the effect on HCC prevention in those patients appears to decline in the long run. Thus we assumed that interferon therapy without attaining SVR had no effect on HCC prevention. Table 4 shows the incidence rates of HCC, as fitted to the crude data by the least squares method. These values were used in modelling of the estimated HCC free survival of individual patients.

\section{HCC free survival}

Using the fitted HCC incidence rates and the age and sex specific death rates, we estimated the lifetime cumulative HCC free survival with or without SVR. Figure l shows an example of a 30 year old male patient with chronic hepatitis $\mathrm{C}$ with stage $\mathrm{F} 3$ fibrosis. The area under the curve indicates the expected HCC free survival and the area between the two curves is the gain in HCC free survival when the patient achieves SVR. The gain, or the area, was calculated to be 12.4 years in this case.

We similarly calculated the gain in HCC free survival under various conditions (see fig 2, table 5). By definition, these values are applicable only after SVR has been achieved. The gain in HCC free survival that can be expected before the virological outcome is known is the product of the value in table 5 and the prior probability of achieving SVR.

The gain in HCC free survival was greater when a patient was younger or fibrosis was more advanced. Judging by the expected gain, indications for treatment are questionable in patients with fibrosis stage F0 or Fl and older than 60 years because they would gain less than one year even if they

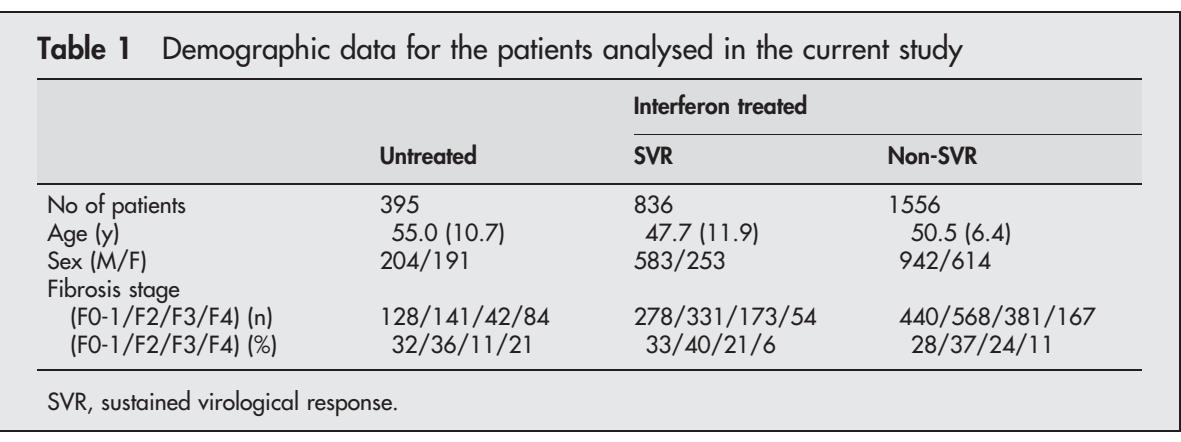


Table 2 Incidence of hepatocellular carcinoma (HCC) and death in the study cohort

\begin{tabular}{lccc} 
& & \multicolumn{2}{l}{ Interferon treated } \\
\cline { 3 - 4 } & Untreated & SVR & Non-SVR \\
\hline No of patients & 395 & 836 & 1556 \\
Follow up (y) & $6.5(2.8)$ & $6.7(3.0)$ & $7.4(2.9)$ \\
HCC development (n) & 67 & 27 & 214 \\
Death $(n)$ & 33 & 11 & 59 \\
With HCC $(n)^{*}$ & 22 & 6 & 8 \\
Hepatic deaths & 4 & 1 & 22 \\
Without HCC (n) & 7 & 4 & \\
Non-hepatic deaths $(n)$ & & & \\
\hline *Includes deaths not directly related to HCC in patients who had developed the cancer. & \\
SVR, sustained virological response. & & &
\end{tabular}

attained SVR. On the other hand, patients with fibrosis stage F3 or F4 and younger than 50 years will gain more than 10 years with SVR, and more than five years even if the probability of attaining SVR is $50 \%$.

Recently, the efficacy of interferon therapy has been improved by the introduction of peginterferon and ribavirin. However, more effective protocols will be accompanied by an increase in cost and incidence of untoward effects. These must be counterbalanced by an increase in expected benefit. While the increase in cost is the same, that in benefit is directly proportional to the values shown in table 5 and differs in each patient. The SVR rate for type lb genotype high viral load infection was approximately $7 \%$ among the current cohort where six months of interferon monotherapy was the main protocol. The combination of peginterferon and ribavirin for 48 weeks, which is still under clinical trials in Japan, is expected to show a response rate of $40 \%$ or better for those patients. This difference $(33 \%)$ corresponds to five years of the gain in HCC free survival in 40 year old patients with fibrosis stage F4 and to approximately one year in 60 year olds with fibrosis stage F2 (one third of the values given in table 5). Although these values are a hypothetical extrapolation, they may be clinically useful in choosing treatment protocols.

\section{Model limitations}

The model described in this article is based on several assumptions. Firstly, we assumed that interferon therapy that failed to achieve SVR had no beneficial effect, as described above, and this may result in underestimation of the benefit. However, the difference cannot be large: a 30 year old male with fibrosis stage $\mathrm{F} 4$ has a gain of 16.59 years instead of 15.98 years, and an 80 year old male with fibrosis stage F0/1 has a gain of 0.18 years instead of 0.15 years if we based the calculations on the incidence observed in the untreated group.

Secondly, we assumed that the benefit of interferon therapy was limited to HCC prevention. This is certainly an underestimation: successful interferon therapy improves liver function and may prevent death from liver failure. Several studies, failing to find an effect on HCC incidence, still indicated improvement in liver function with interferon therapy. ${ }^{29}{ }^{30}$ However, hepatic death without developing HCC was rare in the current cohort; one patient in the SVR group (variceal rupture $(n=1)$ ), eight in the non-SVR group (liver failure $(n=4)$, variceal rupture $(n=3)$, not specified $(\mathrm{n}=1))$, and four in the untreated group (liver failure $(\mathrm{n}=2)$, variceal rupture $(\mathrm{n}=2))$ died of a liver related cause without developing HCC, indicating annual mortality rates of $0.02 \%, 0.08 \%$, and $0.18 \%$, respectively (table 2 ). These values were small relative to the observed incidence of HCC.

Thirdly, we assumed that fibrosis stage remained constant, with the risk of HCC unchanged except for the increment due to aging. This may not be true: in fact, we previously indicated fibrosis progression in untreated patients and amelioration in interferon responders. ${ }^{23}$ However, we did

Table 3 Annual hepatocellular carcinoma $(\mathrm{HCC})$ incidence rates according to age and sex

\begin{tabular}{lllll}
\hline Age (y) & F0/1 & F2 & F3 & F4 \\
\hline SVR, male & & & & \\
$\leqslant 39$ & $0.05 \%(0 / 65)$ & $0.09 \%(0 / 59)$ & $0.16 \%(0 / 14)$ & $0.24 \%(0 / 4)$ \\
$40-49$ & $0.05 \%(0 / 57)$ & $0.09 \%(0 / 66)$ & $0.16 \%(1 / 29)$ & $0.24 \%(0 / 9)$ \\
$50-59$ & $0.39 \%(0 / 38)$ & $0.69 \%(3 / 62)$ & $1.21 \%(5 / 51)$ & $1.86 \%(1 / 18)$ \\
$\quad 60+$ & $0.70 \%(3 / 29)$ & $1.18 \%(3 / 38)$ & $2.01 \%(4 / 35)$ & $3.20 \%(1 / 9)$ \\
SVR, female & & & & \\
$\quad \leqslant 39$ & $0.02 \%(0 / 32)$ & $0.05 \%(0 / 38)$ & $0.10 \%(0 / 7)$ & $0.15 \%(0 / 1)$ \\
$40-49$ & $0.03 \%(0 / 25)$ & $0.05 \%(0 / 23)$ & $0.10 \%(0 / 3)$ & $0.15 \%(1 / 1)$ \\
$50-59$ & $0.23 \%(0 / 20)$ & $0.41 \%(1 / 33)$ & $0.73 \%(1 / 20)$ & $1.12 \%(1 / 6)$ \\
$60+$ & $0.40 \%(0 / 6)$ & $0.71 \%(1 / 18)$ & $1.25 \%(0 / 14)$ & $1.93 \%(1 / 6)$ \\
Non-SVR, male & & & & \\
$\leqslant 39$ & $0.05 \%(0 / 83)$ & $0.13 \%(0 / 72)$ & $0.28 \%(2 / 29)$ & $0.56 \%(0 / 6)$ \\
$40-49$ & $0.35 \%(2 / 85)$ & $1.00 \%(4 / 101)$ & $2.16 \%(7 / 46)$ & $4.26 \%(10 / 32)$ \\
$50-59$ & $0.82 \%(6 / 82)$ & $2.33 \%(19 / 111)$ & $5.06 \%(26 / 74)$ & $10.0 \%(17 / 33)$ \\
$60+$ & $1.03 \%(4 / 36)$ & $2.93 \%(13 / 59)$ & $6.35 \%(17 / 64)$ & $12.5 \%(15 / 29)$ \\
Non-SVR, female & & & & \\
$\leqslant 39$ & $0.02 \%(0 / 37)$ & $0.07 \%(0 / 21)$ & $0.14 \%(0 / 10)$ & $0.29 \%(0 / 2)$ \\
$40-49$ & $0.18 \%(0 / 41)$ & $0.51 \%(2 / 44)$ & $1.10 \%(3 / 18)$ & $2.17 \%(0 / 6)$ \\
$50-59$ & $0.42 \%(1 / 53)$ & $1.19 \%(8 / 96)$ & $2.57 \%(19 / 80)$ & $5.08 \%(5 / 32)$ \\
$60+$ & $0.52 \%(1 / 23)$ & $1.49 \%(11 / 64)$ & $3.23 \%(10 / 60)$ & $6.37 \%(12 / 27)$ \\
\hline
\end{tabular}

The percentages indicate the annual incidence rates fitted by the least squares method using the risk ratios shown in table 4. Numbers in parentheses are the observed events/number at risk in each category.

SVR, sustained virological response; FO-F4, fibrosis stage. 
Table 4 Risk of hepatocellular carcinoma (HCC) development

\begin{tabular}{lll}
\hline & \multicolumn{2}{l}{ Relative risk $(95 \% \mathrm{CI})$} \\
\cline { 2 - 3 } Factor & SVR & Non-SVR \\
\hline Male $v$ female & $1.66(0.67-4.13)$ & $1.97(1.48-2.62)$ \\
Age $(\mathrm{y})$ & 1 & 1 \\
$\leqslant 39$ & 1 & $7.61(1.81-31.93)$ \\
$40-49$ & $7.67(1.69-34.72)$ & $17.84(4.39-72.49)$ \\
$50-59$ & $13.20(2.93-59.53)$ & $22.36(5.48-91.26)$ \\
$60+$ & 1 & 1 \\
Fibrosis stage & $1.76(0.47-6.67)$ & $2.86(1.59-5.13)$ \\
F0/1 & $3.10(0.86-11.26)$ & $6.19(3.50-10.95)$ \\
F2 & $4.78(1.13-20.18)$ & $12.23(6.81-21.95)$ \\
F3 & F4 &
\end{tabular}

not have enough samples to calculate age stratified rates of fibrosis progression. The long term changes in HCC risk in interferon responders have not yet been clearly elucidated. Thus using the incidence rates observed in the seven year observation period was a compromise. All of the assumptions listed here may have underestimated, but not overestimated, the benefit of interferon therapy.

Lastly, we did not analyse the effect of alcohol consumption as there were very few heavy drinkers among the cohort. Alcohol is one of the major risk factors for HCC development and liver failure. The merit of successful interferon therapy may be greater in drinkers if they wish to continue alcohol. However, we recommend abstention to chronic hepatitis C patients whether or not they receive antiviral therapy.

\section{DISCUSSION}

To date, large scale cohort studies conducted in Japan, including ours, have unanimously indicated that by far the most important sequela of chronic hepatitis C is HCC development, and that interferon therapy significantly reduces its incidence. In contrast, several studies performed in Western countries found that HCC was less common, and interferon therapy did not have significant effects. The reason for this discrepancy has not been clarified. In this study, we showed that the risk of HCC substantially increased with age when patients of the same sex and fibrosis stage were compared (table 2). The prevalence of HCV infection in Japan is highly skewed to the older generations, and this may partly explain the high incidence of HCC in Japan. If this is the case, HCC incidence will increase substantially in Western countries in the near future, as it did in Japan in the 1980s.

The clinical importance of interferon therapy should be measured in terms of its efficacy in HCC prevention, at least in countries where HCC is the predominant complication of

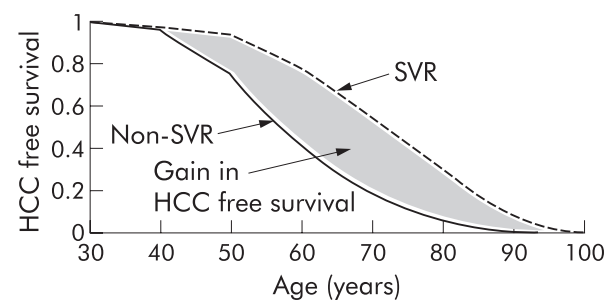

Figure 1 Gain in hepatocellular carcinoma (HCC) free survival by interferon therapy. The case of a 30 year old male patient with fibrosis stage F3. Cumulative HCC free survival curves were determined based on the patient specific HCC incidence rates and age and sex specific death rates in case of sustained virological response (SVR) and non-SVR. The area surrounded by the two curves indicates the gain in HCC free survival obtained by achieving SVR.
HCV infection. A popular indicator of efficacy of therapy in preventing a disease is the number (of patients) needed to treat (NNT), which is identical to the inverse of absolute risk reduction. Mathematically, NNT for one decrement in HCC development during a lifetime is equivalent to the life expectancy divided by the gain in HCC free survival. Supposing that the SVR rate is $100 \%$, NNT is $3.92(48.7 /$ 12.4; table 5) for a Japanese 30 year old male patient with fibrosis stage F3. This value should be divided by the expected SVR rate if the outcome is not known. As NNT is directly proportional to life expectancy, older patients have smaller values for NNT, indicating "higher efficacy", if the gain in HCC free survival is the same. This may not be intuitive for individualised consideration of indications for treatment.

Several authors have performed cost effective analyses of interferon therapy for chronic hepatitis $\mathrm{C}$ based on the Markov model..$^{31-35}$ In fact, our current model can be considered as a simplified Markov model where a chronic hepatitis C patient either achieves or does not achieve SVR with interferon therapy, and has the corresponding risk of HCC thereafter. Also, the HCC free survival is equivalent to the gain in quality adjusted life year, where a year before HCC development scores 1 and one after it scores 0. Owing to those simplifications, the model is not dependent on assumptive parameters but on observed data.

In conclusion, by using data obtained in a real cohort, we established an indicator of the benefit of interferon therapythe gain in HCC free survival. This indicator may be useful in assessing the indications for interferon therapy and in selecting the best treatment protocol for individual patients.
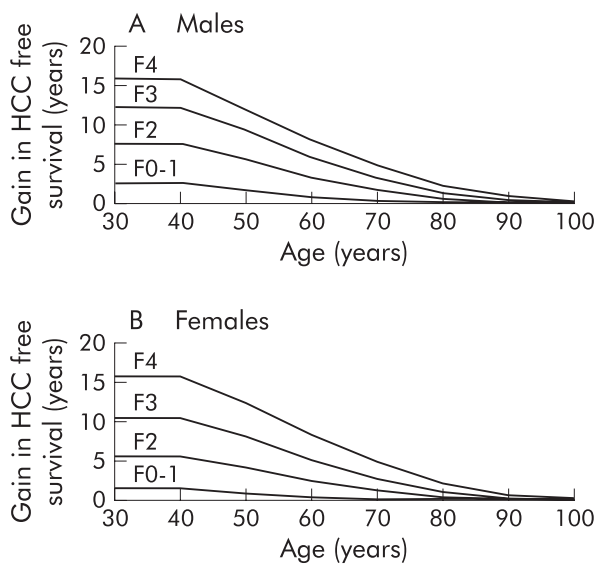

Figure 2 Gain in hepatocellular carcinoma (HCC) free survival by sustained virological response as a function of age and fibrosis stage. 
Table 5 Gain in hepatocellular carcinoma (HCC) free survival by sustained virological response as a function of age and fibrosis stage

\begin{tabular}{ccccrc}
\hline Age $(\mathbf{y})$ & Life expectancy & F0/1 & F2 & F3 & F4 \\
\hline Males & & & & & \\
30 & 48.7 & 2.48 & 7.66 & 12.40 & 15.98 \\
40 & 39.1 & 2.52 & 7.68 & 12.41 & 15.96 \\
50 & 29.9 & 1.68 & 5.75 & 9.45 & 12.14 \\
60 & 21.4 & 0.84 & 3.38 & 5.95 & 8.14 \\
70 & 14.0 & 0.40 & 1.70 & 3.26 & 4.98 \\
80 & 8.0 & 0.15 & 0.67 & 1.40 & 2.38 \\
Females & & & & & \\
30 & 55.3 & 1.45 & 5.60 & 10.52 & 15.73 \\
40 & 45.5 & 1.46 & 5.61 & 10.51 & 15.69 \\
50 & 36.0 & 0.93 & 4.24 & 8.17 & 12.44 \\
60 & 26.9 & 0.44 & 2.52 & 5.17 & 8.39 \\
70 & 18.2 & 0.22 & 1.30 & 2.81 & 4.95 \\
80 & 10.6 & 0.08 & 0.52 & 1.18 & 2.24 \\
\hline
\end{tabular}

Expressed in years, life expectancy was that in the Japanese general population in 2000. The gain in HCC free survival was the difference in expected cumulative HCC free survival with and without attaining a sustained virological response.

\section{ACKNOWLEDGEMENTS}

This study was supported, in part, by a Grant-in-Aid from the Ministry of Health, Welfare, and Labour of Japan.

\section{Authors' affiliations}

H Yoshida, R Tateishi, Y Shiratori*, M Omata, Department of Gastroenterology, University of Tokyo, Tokyo, Japan

Y Arakawa, Third Department of Internal Medicine, Nihon University, Tokyo, Japan

M Sata, Second Department of Internal Medicine, Kurume University, Fukuoka, Japan

S Fujiyama, Third Department of Internal Medicine, Kumamoto University, Kumamoto, Japan

S Nishiguchi, Third Department of Internal Medicine, Osaka City University, Osaka, Japan

H Ishibashi, Department of Clinical Research, National Nagasaki Medical Centre, Nagasaki, Japan

G Yamada, Centre of Liver Disease, Kawasaki Hospital Kawasaki Medical School, Okayama, Japan

O Yokosuka, First Department of Internal Medicine, Chiba University, Chiba, Japan

*Present address: Department of Gastroenterology, Hepatology, and Infectious Diseases, Okayama University, Okayama

\section{REFERENCES}

1 Yamada G, Tanaka E, Miura T, et al. Epidemiology of genotypes of hepatitis C virus in Japanese patients with type $C$ chronic liver diseases: a multi-institution analysis. J Gastroenterol Hepatol 1995; 10:538-45.

2 Yano $M$, Kumada $\mathrm{H}$, Kage $\mathrm{H}$, et al. The long-term pathological evolution of chronic hepatitis C. Hepatology 1996;23:1334-40

3 Moriya T, Koyama T, Tanaka J, et al. Epidemiology of hepatitis $\mathrm{C}$ virus in Japan. Intervirology 199:42:153-8.

4 Wasley A, Alter MJ. Epidemiology of hepatitis C: geographic differences and temporal trends. Semin Liver Dis 2000;20:1-16.

5 Tanaka Y, Hanada K, Mizokami M, et al. Inaugural article: A comparison of the molecular clock of hepatitis $C$ virus in the United States and Japan predicts that hepatocellular carcinoma incidence in the United States will increase over the next two decades. Proc Natl Acad Sci U S A 2002;99:15584-9.

6 Kuo G, Choo QL, Alter HJ, et al. An assay for circulating antibodies to a major etiologic virus of human non-A, non-B hepatitis. Science 1989:244:362-4.

7 Choo QL, Kuo G, Weiner AJ, et al. Isolation of a cDNA clone derived from a blood-borne non-A, non-B viral hepatitis genome. Science 19789;244:359-62.

8 Hoofnagle JH, Mullen KD, Jones DB, et al. Treatment of chronic non- $\mathrm{A}$, non-B hepatitis with recombinant human alpha interferon. A preliminary report. N Engl J Med 1986;315:1575-8.

9 Di Bisceglie AM, Martin P, Kassianides C, et al. Recombinant interferon alfa therapy for chronic hepatitis C. A randomized, double-blind, placebocontrolled trial. N Engl J Med 1989:321:1506-10.

10 Omata $M$, Yokosuka O, Takano S, et al. Resolution of acute hepatitis $C$ after therapy with natural beta interferon. Lancet 1991;338:914-15.

11 Nishiguchi S, Kuroki T, Nakatani S, et al. Randomised trial of effects of interferon-alpha on incidence of hepatocellular carcinoma in chronic active hepatitis C with cirrhosis. Lancet 1995;346:1051-5.
12 Mazzella G, Accogli E, Sottili S, et al. Alpha interferon treatment may prevent hepatocellular carcinoma in HCV-related liver cirrhosis. J Hepatol 1996:24:141-7.

13 Kasahara A, Hayashi N, Mochizuki K, et al. Risk factors for hepatocellular carcinoma and its incidence after interferon treatment in patients with chronic hepatitis C. Osaka Liver Disease Study Group. Hepatology 1998:27:1394-402.

14 Imai Y, Kawata S, Tamura S, et al. Relation of interferon therapy and hepatocellular carcinoma in patients with chronic hepatitis C. Osaka Hepatocellular Carcinoma Prevention Study Group. Ann Intern Med 1998:129:94-9.

15 Yoshida H, Shiratori Y, Moriyama M, et al. Interferon therapy reduces the risk for hepatocellular carcinoma: national surveillance program of cirrhotic and non-cirrhotic patients with chronic hepatitis C in Japan. IHIT Study Group. Inhibition of Hepatocarcinogenesis by Interferon Therapy. Ann Intern Med 1999:131:174-81.

16 Reichard O, Norkrans G, Fryden A, et al. Randomised, double-blind, placebo-controlled trial of interferon alpha- $2 \mathrm{~b}$ with and without ribavirin for chronic hepatitis C. The Swedish Study Group. Lancet 1998;351:83-7.

17 Poynard T, Marcellin P, Lee SS, et al. Randomised trial of interferon alpha $2 b$ plus ribavirin for 48 weeks or for 24 weeks versus interferon alpha $2 \mathrm{~b}$ plus placebo for 48 weeks for treatment of chronic infection with hepatitis $C$ virus. International Hepatitis Interventional Therapy Group (IHIT). Lancet 1998;352:1426-32.

18 McHutchison JG Gordon SC, Schiff ER, et al. Interferon alfa-2b alone or in combination with ribavirin as initial treatment for chronic hepatitis $C$. Hepatitis Interventional Therapy Group. N Engl J Med 1998;339:1485-92.

19 Zeuzem S, Feinman SV, Rasenack J, et al. Peginterferon alfa-2a in patients with chronic hepatitis C. N Engl J Med 2000;343:1666-72.

20 Manns MP, Mctutchison JG, Gordon SC, et al. Peginterferon alfa- $2 b$ plus ribavirin compared with interferon alfa- $2 \mathrm{~b}$ plus ribavirin for initial treatment of chronic hepatitis C: a randomised trial. Lancet 2001;358:958-65.

21 Lindsay KL, Trepo C, Heintges T, et al. A randomized, double-blind trial comparing pegylated interferon alfa- $2 b$ to interferon alfa- $2 b$ as initial treatment for chronic hepatitis C. Hepatology 2001:34:395-403.

22 Fried MW, Shiffman ML, Reddy K, et al. Peginterferon alfa-2a plus ribavirin for chronic hepatitis C virus infection. N Engl J Med 2002;347:975-82.

23 Shiratori Y, Imazeki F, Moriyama M, et al. Histologic improvement of fibrosis in patients with hepatitis $C$ who have sustained response to interferon therapy. Ann Intern Med 2000;132:517-24.

24 Yoshida H, Arakawa Y, Sata $M$, et al. Interferon therapy prolonged life expectancy among chronic hepatitis $C$ patients. Gastroenterology 2002;123:483-91.

25 Management of Hepatitis C. NIH Consensus Statement Online 1997 Mar 2426;15(3):1-41. http://odp.od.nih.gov/consensus/cons/105/ 105_statement.htm.

26 Desmet VJ, Gerber M, Hoofnagle JH, et al. Classification of chronic hepatitis: diagnosis, grading and staging. Hepatology 1994;19:1513-20.

27 The 19th National Complete Life Table (2000). The Ministry of Health Welfare and Labour of Japan. http://www.mhlw.go.jp/toukei/saikin/hw/ life/19th/.

28 Shindo $\mathrm{M}$, Hamada K, Oda Y, et al. Long-term follow-up study of sustained biochemical responders with interferon therapy. Hepatology 2001;33:1299-302.

29 Fattovich G, Giustina G, Degos F, et al. Effectiveness of interferon alfa on incidence of hepatocellular carcinoma and decompensation in cirrhosis type C. European Concerted Action on Viral Hepatitis (EUROHEP). J Hepatol 1997; 27:201-5.

30 Niederau C, Lange S, Heintges T, et al. Prognosis of chronic hepatitis C: results of a large, prospective cohort study. Hepatology 1998;28:1687-95.

31 Bennett WG, Inove Y, Beck JR, et al. Estimates of the cost-effectiveness of a single course of interferon-alpha $2 \mathrm{~b}$ in patients with histologically mild chronic hepatitis C. Ann Intern Med 1997;127:855-65. 
32 Younossi ZM Singer ME, McHutchison JG et al Cost effectiveness of interferon alpha2b combined with ribavirin for the treatment of chronic hepatitis C. Hepatology 1999;30:1318-24.

33 Stein K, Rosenberg W, Wong J. Cost effectiveness of combination therapy for hepatitis C: a decision analytic model. Gut 2002;50:253-8.
34 Buti M, Medina M, Casado MA, et al. A cost-effectiveness analysis of peginterferon alfa- $2 b$ plus ribavirin for the treatment of naive patients with chronic hepatitis C. Aliment Pharmacol Ther 2003;17:687-94.

35 Siebert U, Sroczynski G, Rossol S, et al. Cost effectiveness of peginterferon alpha- $2 \mathrm{~b}$ plus ribavirin versus interferon alpha- $2 \mathrm{~b}$ plus ribavirin for initial treatment of chronic hepatitis C. Gut 2003;52:425-32.

\section{GI SNAPSHOT}

\section{Answer}

From question on page 386

Figure 1 shows a water soluble contrast enema, which revealed obstruction at the rectosigmoid, apparently caused by an extrinsic pelvic mass.

An emergency laparotomy for large bowel obstruction was carried out. At operation she was found to have a dilated small and large bowel with a cut off at the rectosigmoid. The cause of the obstruction was a hugely enlarged fibroid uterus which was incarcerated in the pelvis. A subtotal hysterectomy was performed to relieve the obstruction. The compressed large bowel was found to be healthy and did not necessitate resection. She eventually made a complete recovery, enjoying normal bowel function.

Histology confirmed a hugely enlarged uterus distorted by a single $11 \times 9 \times 7.5 \mathrm{~cm}$ leiomyoma with no evidence of dysplasia or malignancy.

Gynaecological disease and its treatment is a relatively common cause of bowel obstruction. The commonest cause is gynaecological malignancy, particularly that of the ovaries and complications of treatment such as adhesions and radiotherapy.

Leiomyomas or fibroids are benign smooth muscle tumours of the uterine myometrium and are a common condition in women especially over the age of 40 years. Small leiomyomas are present in more than $20 \%$ of women over the age of 40 years and usually remain asymptomatic. However, bowel obstruction secondary to benign uterine leiomyomas may occur, albeit rarely.

This should be considered early in female patients and if rapid sustained resolution of the clinical features of obstruction does not occur, the condition should be treated aggressively with surgical intervention. 